\title{
Simvastatin Enhances Clinical Response to Sulfadoxine-Pyrimethamine in Falciparum Malaria NN Nwobodo ${ }^{1}$, PO Okonkwo ${ }^{2}$
}

\begin{abstract}
Background: Doubts have been raised concerning the therapeutic efficacy of sulfadoxine-pyrimethamine due to poor clinical response necessitating the need for continuous monitoring and further advocating for the replacement of sulfadoxine-pyrimethamine with suitable alternatives. Statins are known to down regulate biosynthesis of dolichol and isoprenoid pyrophosphate, inhibiting in vitro growth of Plasmodium falciparum.

Objectives: This study was aimed at evaluating the clinical response of simvastatin plus sulfadoxinepyrimethamine combination as compared to sulfadoxine-pyrimethamine alone in the chemotherapy of malaria.

Methods: Malaria patients $(n=60)$ confirmed by thick blood film and immunological tests were selected and informed written consent obtained. Patients were categorized into simvastatin plus sulfadoxine-pyrimethamine (test) and sulfadoxine-pyrimethamine alone (control). The University of Nigeria Teaching Hospital Research Ethics Committee reviewed the proposal and provided ethical clearance certification (NHREC/05/01/2008B). The WHO was adopted in the assessment of clinical response and patients followed up on days D0, D3, D7, D14 and D28 post-treatment. The analysis of data was done using GraphPad Prism 4.0 and data presented in tabular and graphical forms.
\end{abstract}

Results: The mean early treatment failure given as $9.2 \pm 0.26 \%$ in the test group was significantly decreased relative to $20.5 \pm 0.26 \%$ in the control; whereas mean late treatment failure given as $10.3 \pm 0.15 \%$ in the test group was reported relative to $23.6 \pm 0.22 \%$ in the control. There was statistically significant increase in adequate clinical and parasitological response given as $77.5 \pm 0.59 \%$ in the test group relative to $55.9 \pm 0.49 \%$ in the control. The mean parasite clearance time and fever clearance time given in the test group as $4.4 \pm 0.2$ days and $55.3 \pm 2.6$ hours were significantly reduced relative to $9.8 \pm 0.2$ days and $87.4 \pm 4.5$ hours recorded in the control. The clinical clearance rate given as $82.3 \pm 0.8 \%$ was significantly increased in the test group relative to $60 \pm 1.3 \%$ recorded in controls. A statistically significant decrease was recorded in the recrudescence rate given as $19.1 \pm 0.14 \%$ in the test group relative to $39 \pm 0.22 \%$ recorded in the control.

Keywords: Clinical response, Fever clearance, Malaria, Parasite clearance, Plasmodium falciparum, Recrudescence, Simvastatin, Sulfadoxine-pyrimethamine, Treatment failure.

\footnotetext{
${ }^{1}$ Department of Pharmacology and Therapeutics, Ebonyi State University, Abakaliki, Nigeria ${ }^{2}$ Department of Pharmacology and Therapeutics, University of Nigeria

Correspondence: Dr Nwobodo,Division of Clinical Pharmacology and Translational MedicineDepartment of Pharmacology \& Therapeutics Faculty of Clinical Medicine Ebonyi State University PMB 53, Abakaliki, Nigeria, E-mail: nnwobodo@yahoo.com
} 
Conclusion: The enhancement in clinical response in this study is attributable to modulating influence of statin treatment. Thus, the 3-HMG-CoA reductase inhibitor, simvastatin, is a potential candidate for combinational anti-malarial chemotherapy. 


\section{INTRODUCTION}

A study raised doubts about the therapeutic efficacy of sulfadoxine-pyrimethamine due to poor clinical response, necessitating the need for continuous monitoring and further advocated the replacement of sulfadoxine-pyrimethamine with suitable alternatives (1). Indeed, the therapeutic life of sulfadoxine-pyrimethamine was predicted to last for only five years following its introduction in Africa as alternative to chloroquine, due to high transmission rates of malaria. Sulfadoxine-pyrimethamine has been recommended by World Health Organisation (WHO), for intermittent preventive therapy (IPT) in pregnancy, particularly in the disease endemic zones (2). A study hypothesized that combination of molecular and epidemiological factors may be implicated in the clinical efficacy of sulfadoxine- pyrimethamine (3). The declining clinical efficacy and parasite clearance rates, all point to the dire need for novel effective treatment of malaria infection. A number of mechanisms acting through various organ systems are involved in the pathophysiology of malaria infection (4). Statins are known to down regulate biosynthesis of dolichol and isoprenoid pyrophosphate, inhibiting in vitro growth of Plasmodium falciparum (5). A study revealed that the clinical benefit of statins could be attributed not onl;y to improvement in endothelial function, but a significant reduction in anti-inflammatory effects(6). Simvastatin has been shown to reduce tumor necrosis factor- $\alpha$ (TNF- $\alpha$ ), Interleukin-1(IL-1) and proinflammatory cytokines $(7,8)$. The production of TNF- $\alpha$ and IL-1 is induced by glycosylphosphatidylinositol (GPI) produced by Plasmodium falciparum, leading to TNF- $\alpha$ induced weight loss in malaria infection $(9,10)$. Consequently, the present study was aimed at evaluating the clinical response of simvastatin plus sulfadoxine-pyrimethamine (test group) combination as compared to sulfadoxine-pyrimethamine alone (control group) in the chemotherapy of malaria. 


\section{METHODS}

Subjects: The subjects selected for this stuidy were patients in attendance at primary health facilities $(n=60)$ suffering from malaria infection, diagnosed using thick blood films and confirmed by immunological test $\left(\right.$ Paracheck $\left.\mathrm{PI}^{\circledR}\right)$. A rapid qualitative two site sandwich immunochromatographic dipstick assay, (Paracheck $\mathrm{PI}^{\circledR}$ ), was employed for the determination of Plasmodium falciparum specific histidine rich protein-2 (PfHRP-2) in whole blood samples. This was in view of the fact that classical method of diagnosis by microscopy involving examination of thin and thick blood smears was time consuming and prone to false negative readings.

Study Design: Formal written documentation was employed in obtaining informed consent after adequate explanation of the purpose of study, type of treatment to be administered and clarification of any likely adverse effects or complication that may arise in the course of treatment. Patients within the age range 16 to 65 years inclusive attending eight primary health facilities within Asu Nkanu Local Health Authority in Nkanu East Local Government Area of Enugu State, Nigeria were selected for the study. The subject's physical condition and presence of any confounding ailment were ascertained following routine clinical clerkship and examination including body weight measurement and axillary temperature. Subjects were randomised into test and control groups using a table of random numbers statistically generated. The principal investigator, microscopist, field supervisor, field assistants, medical officer, nurses and all other participants in the study did not have any prior knowledge of the patients' medical records nor the treatment group to which each subject was assigned. The Health Research Ethics Committee, University of Nigeria Teaching Hospital Ituku-Ozalla, Nigeria provided ethical clearance certification (Ref: NHREC/05/01/2008B) in line with principles guiding human experimentation as enumerated in the Declaration of Helsinki by the World Medical Association General Assembly as last amended (Seoul 2008); 
while Enugu State Ministry of Health, Nigeria provided approval for this study. Sulfadoxinepyrimethamine (Fansidar ${ }^{\circledR}$ from Swiss Pharma, Lagos-Nigeria) was given as stat dose of $25 \mathrm{mg} / \mathrm{kg}$ for the Sulfadoxine component and $1.25 \mathrm{mg} / \mathrm{kg}$ for the other component Pyrimethamine. Each tablet of Sulfadoxine-Pyrimethamine contains 500mg Sulfadoxine and 25mg Pyrimethamine. Simvastatin (Simvor ${ }^{\circledR}$ from Ranbaxy Laboratories, Dewas, Nigeria) was given orally in the dosage $0.6 \mathrm{mg} / \mathrm{kg} / \mathrm{d}$ only in the evening for 3 consecutive days. The control group received Sulfadoxine-pyrimethamine only in same dose as test group. Patients who presented with treatment failure or recrudescence were salvaged with ArtemetherLumefantrine (Coartem $^{\circledR}$ from Novartis Pharma AG, Basel-Switzerland); and eventually withdrawn from the study. The Artemether component was given as $3.2 \mathrm{mg} / \mathrm{kg} / \mathrm{d}$ while the Lumefantrine as $19.2 \mathrm{mg} / \mathrm{kg} / \mathrm{d}$ respectively in two divided doses for 3 days. Baseline monitoring of liver function tests was done before commencement and in the course of therapy. Elevation of serum transaminase activity up to three times normal level will result to discontinuation of simvastatin.

Assessment of Response: The patients were followed up on days D0, D3, D7, D14 and D28. The World Health Organisation (WHO) criteria were applied in the categorization of therapeutic response as follows:

○ Early Treatment Failure (ETF): Development of danger signs of severe malaria on D1-D3 in the presence of parasitemia. Parasitemia on D2 higher than D0 count irrespective of axillary temperature. Parasitemia on D3 with axillary temperature $\geq$ $37.5^{\circ} \mathrm{C}$

- Late Treatment Failure (LTF): Development of danger signs of severe malaria after D3 in the presence of parasitemia, without previously meeting any of the criteria of early treatment failure. Presence of parasitemia and axillary temperature $\geq 37.5^{\circ} \mathrm{C}$ on 
any day from D4 to D14, without previously meeting any of the criteria of early treatment failure.

○ Late Parasitological Failure (LPF): Presence of parasitemia on D28 and axillary temperature $<37.5^{\circ} \mathrm{C}$ without previously meeting any of the criteria of early treatment failure or late treatment failure.

○ Adequate Clinical and Parasitological Response (ACPR): Absence of parasitemia on D14 irrespective of axillary temperature without previously meeting any of the criteria of early treatment failure or late treatment failure

o Fever Clearance Time (FCT): The time taken from anti-malarial drug administration until axillary temperature falls below $37.4^{\circ} \mathrm{C}$ and remains at that value for 72 hours.

o Parasite Clearance Time (PCT): The time taken from anti-malarial drug administration until no patent parasitemia is detected.

- Clinical Clearance Rate (CCR): The proportion of subjects with full resolution of signs and symptoms of malaria on D14.

○ Recrudescence Rate (RR): The proportion of subjects in which there is incomplete clearance of parasitemia on D14 and D28 of follow-up.

Statistical Analysis: The analysis of data was facilitated using Graphpad Prism version 4.0 (GraphPad Software, Inc., La Jolla, CA, USA) statistical software and data presented in tabular and graphical forms. Statistical test of significance ascertained using two-tailed Student $t$-test, assuming $\mathrm{p}<0.05$ considered significant at $95 \%$ confidence interval. 


\section{RESULTS}

Table 1 depicts the baseline characteristics of test and control groups at presentation.

Table 2 shows mean values of treatment failure in patients treated with sulfadoxinepyrimethamine and simvastatin (test) and those treated with sulfadoxine-pyrimethamine alone (control). Table 3 clearly depicts the mean values of Parasite Clearance Time (PCT), Fever Clearance Time (FCT), Clinical Clearance Rate (CCR), Recrudescence Rate (RR) and Cure Rate (CR) in both test and control groups. The mean geometric parasite densities of test and control groups on follow-up days D0, D3, D7, D14 and D28 are as shown in Figure 1 Figure 1 depicts the mean geometric parasite densities of the test and control groups on follow-up days D0, D3, D7, D14 and D28.

\section{DISCUSSION}

Results revealed a statistically significant difference $(\mathrm{p}<0.05)$ in declining parasitemia days 0 , 3,7,14 and 28 between test and control groups as shown in Figure 1. A study reported values of $5 \%$ and $18 \%$ as proportion of initial parasitemia at days 14 and 28 respectively (11) in patients treated with sulfadoxine-pyrimethamine alone. The same study reported that $77 \%$ of the patients achieved complete parasite clearance by day 3 after enrollment and treatment with sulfadoxine-pyrimethamine. A study had emphasized the use of mean geometric gametocyte densities in assessment of response to sulfadoxine-pyrimethamine(12). That study attempted to evaluate the effects of sulfadoxine-pyrimethamine on gametocyte generation during treatment to determine whether or not sulfadoxine-pyrimethamine will influence the generation of gametocytemia and to evaluate peripheral young gametocytes $(\mathrm{PYG})$ as an indicator of response to therapy. 
It has already been documented that sulfadoxine-pyrimethamine may or may not enhance gametocyte carriage during treatment of acute falciparum infections 13,14). Notwithstanding, that the presence of gametocytes in peripheral blood after anti-malarial treatment was no proof of viability, their generation was required for the transmission of the infection from the vertebrates to the anopheline host. Instructively, it was not clear whether the enhancement or non-enhancement of gametocyte production by sulfadoxine-pyrimethamine will be influenced by its use in combination with statins. However, it has been shown that peripheral young gametocytes (PYG) were not an indicator of response to sulfadoxine-pyrimethamine, since both sulfadoxine-pyrimethamine sensitive and resistant infections generated peripheral young gametocytes. Thus, the non-determination of gametocyte carriage status may not necessarily be considered a limitation in the present study.

This study reported a relatively higher therapeutic failure in the control compared to test group treated with sulfadoxine-pyrimethamine as shown in Table 2. A total treatment failure of $36.4 \%$ was reported in a study in South-East Nigeria (15). Again, there was relatively better clinical response in the test group compared to control as evidenced by ACPR (Adequate Clinical and Parasitological Response) of $77.5 \%$ and $55.9 \%$ respectively. The total treatment failure of $19.5 \%$ in the test group treated with sulfadoxine-pyrimethamine was considered below the $25 \%$ limit recommended for centers in the high endemicity of malaria transmission. The early treatment failure (ETF) rate of $9.2 \%$ reported in the test group was still below the $10 \%$ margin recommended for change in first line anti-malarial therapy as compared to $20.5 \%$ in the control. Consequently, the continuous use of sulfadoxine-pyrimethamine will create an apparent well-being but engender a tendency to chronic malaria morbidity in the study population particularly the control.

The World Health Organization has consistently recommended to malaria control programs in Africa to rely more on clinical rather than parasitological response in assessment 
of anti-malarial efficacy. The widespread decline in chloroquine efficacy three decades ago, then necessitated many African countries to adopt sulfadoxine-pyrimethamine as first line therapy in treatment of uncomplicated falciparum malaria. However, progressive decline in sensitivity of Plasmodium falciparum to sulfadoxine-pyrimethamine had since been reported $(16,17)$.

The mean fever clearance time (FCT) in this study is as given in Table 3. This was as compared to 33.6 hours reported in an earlier study (18). However, recent studies in SouthWest and South-East Nigeria revealed fever clearance time of 52.8 and 74.6 hours respectively in subjects treated with sulfadoxine-pyrimethamine alone, which corroborated results in the present study $(12,19)$. The fever clearance time of 33.6 hours and parasite clearance time of 3.9 days obtained in a previous study differed significantly from the values obtained in control group treated with sulfadoxine-pyrimethamine in the present study. This was not surprising as the previous study was carried out over a decade ago when clinical response to sulfadoxine-pyrimethamine was far more reliable.

The mean parasite clearance time (PCT) reported for this study is as shown in Table 3. This was as compared to a recent study which reported parasite clearance time of 6.1days in subjects treated with sulfadoxine-pyrimethamine alone (19). The time to $50 \%$ and $90 \%$ clearance $\left(\mathrm{PC}_{50}\right.$ and $\left.\mathrm{PC}_{90}\right)$ for the said study were given as 0.82 and 1.12 days respectively. It further highlighted that indices of therapeutic response estimated by conventional methods, that is time to $50 \%$ or $90 \%$ reduction of parasitemia and parasite clearance time, were sufficiently higher than those derived from the corresponding functional viability estimate $e x$ vivo for sulfadoxine-pyrimethamine. However, the conventional method employed in the present study was unable to differentiate viable from non-viable circulating parasites (20).

The recrudescence rates reported in this study were $19.1 \%$ and $39 \%$ in the test and control groups respectively treated with sulfadoxine-pyrimethamine. However, clinical 
clearance rate of $82.3 \%$ and $60 \%$ were reported in the present study for the test and control groups respectively. The above values correlated with cure rate of $74.5 \%$ and $55.9 \%$ reported for the test and control groups respectively in this study.

The trophozoite stage of malaria parasite which is analogous to $G_{1}$ phase of the mammalian cell cycle, is susceptible to simvastatin; and the cytostatic effects observed related to $G_{1}$ phase. The $G_{0}$ phase of mammalian cell cycle is related to the early ring stage which can develop in vitro in the absence of serum components (21). The re-initiation of parasite development shows strong similarities to the proliferation of mammalian cells in the $\mathrm{G}_{0}$ phase after stimulation with lipoproteins(22).

The traversal of the cellular barrier between liver cells and the circulatory system is a crucial step in malaria infection. Hence, targeting the liver with statins could be a very effective strategy for novel malaria treatment and prevention. In conclusion, the enhancement of clinical response to sulfadoxine-pyrimethamine in the present study is attributable to the modulating influence of the HMG-CoA reductase inhibitor, simvastatin.

\section{ACKNOWLEDGMENTS}

I wish to acknowledge the immense contribution of Mr. M.O. Offu and the entire staff of 8 primary health facilities in the study site within Asu-Nkanu Local Health Authority. Indeed, worthy of mention is Prof. Eddy Nwobodo, formerly, Dean, Faculty of Basic Medical Sciences, Nnamdi Azikiwe University for his useful advice and contribution. The selfless contribution of Dr. Nick C. Obitte, Lecturer, Department of Pharmaceutical Technology, University of Nigeria, Nsukka is highly appreciated. I acknowledge with gratitude the selfless assistance of Mr. E.A. Aguodoh, of the Department of Hematology, University of Nigeria 
Teaching Hospital, Ituku-Ozalla and Mr. Chuks Ihionu, Director, McChuks Laboratory, Enugu.

\section{DECLARATIONS}

Authors' contributions: NNN and POO conceived and designed the study. NNN conducted the study and gathered data. Both authors analysed and interpreted the data. NNN drafted the manuscript which was critically reviewed by POO. Both authors read and approved the final version of the manuscript before submission.

Conflict of Interest: None disclosed.

Funding: None.

Ethical Clearance: Obtained from University of Nigeria Teaching Hospital, Health Research Ethics Committee (Ref: NHREC/05/01/2008B) 


\section{REFERENCES}

1. Warsame M, Abdillahi A, Duale NO, Ismail A N, Hassan AM, Mohamed A et al. Therapeutic efficacy of chloroquine and sulfadoxine-pyrimethamine against Plasmodium falciparum infection in Somalia. Bulletin of World Heath Organisation 2002; 80: 704-708.

2. WHO Expert Committee on Malaria $20^{\text {th }}$ Report. WHO technical report series; 892 , pp 42-43, 2000.

3. Plowe CV, Kublin JG, Dzinjalamala FK, Kamwendo DS, Mukadam RAG, Chimpeni $P$ et al. Sustained clinical efficacy of sulfadoxine pyrimethamine for uncomplicated falciparum in Malawi after 10 years as first line treatment: five year prospective study. BMJ 2004; 328(7439): 545.

4. Clark IA, Awburn MM, Whitten RO, Harper CG, Liomba NG, Molyneux ME et al. Tissue distribution of migration inhibitory factor and inducible nitric oxide synthase in falciparum malaria and sepsis in African children. Malaria Journal 2003; 2: 6.

5. Couto AS, Kimura EA, Peres VJ, Uhrig ML, Katzin AM. Active isoprenoid pathway in the intra-erythrocytic stages of Plasmodium falciparum: presence of dolichols of 11 and 12 isoprene units. Biochem J 1999; 341: 629-637.

6. Tirona RG, Kim RB. Pharmacogenomics of drug transporters. In: Licinio J, Wong ML, editors. Pharmacogenomics: the search of individualized therapeutics. Weinheim: Wiley VCH. pp. 179-214, 2002.

7. Solheim S, Seljeflot I, Arnesen H, Eritsland J, Eikvar L. Reduced levels of TNF alpha in hypercholesterolemic individuals after treatment with pravastatin for 8 weeks. Atherosclerosis 2001; 157: 411-415. 
8. Ferro D, Violi F. Simvastatin inhibits the monocyte expression of pro-inflammatory cytokines in patients with hypercholesterolemia. J Am Coll Cardiol 2000; 36: $427-$ 431.

9. Urban BC, Ferguson DJP, Pain A, Willcox N, Plebanski M, Austyn JM et al. Plasmodium falciparum-infected erythrocytes modulate the maturation of dendritic cells. Nature 1999; 400: 73-77.

10. Schofield L, Hackett F. Signal transduction in host cells by glycosylphosphatidylinositol toxins of malaria parasites. J Exp Med 1993; 177: 145-153.

11. Nwanyanwu OC, Ziba C, Kazembe PN, Gamadzi G, Gondwe J, Redd SC. The effect of oral iron therapy during treatment for Plasmodium falciparum malaria with sulfadoxine-pyrimethamine on Malawian children under 5 years of age. Annals of Tropical Medicine and Parasitology 1996; 90(6): 589-595.

12. Sowunmi A, Adedeji AA, Gbotosho GO, Fateye BA, Happi TC. Effects of sulfadoxine-pyrimethamine, chloroquine plus chlorpheniramine and amodiaquine plus sulfadoxine-pyrimethamine on gametocytes during and after treatment of acute uncomplicated malaria in children. Mem Inst Oswaldo Cruz 2006; 101(8): 887-895.

13. Puta C, Mayando C. Enhanced gametocyte production in fansidar-treated Plasmodium falciparum malaria patients: implications for malaria transmission control programmes. Trop Med Inter Hlth 1997; 2: 227-229.

14. Hogh B, Thompson R, Hetzel C, Knise NA, Jones I, Dgedge M et al. Specific and non-specific responses to Plasmodium falciparum blood-stage parasites and observations on the gametocytemia in school children living in a malaria endemic area of Mozambique. Am J Trop Med Hyg 1995; 52: 50-59.

15. Okafor HU, Oguonu T. Comparative evaluation of the efficacy of chloroquine and sulfadoxine-pyrimethamine in the treatment of uncomplicated malaria in children 
under five years in a rural population of Enugu State. Journal of College of Medicine 2004; 9(2): 77-81.

16. Falade CO, Salako LA, Sowunmi A, Oduola AMJ, Larcier P. Comparative efficacy of halofanthrine, chloroquine and sulfadoxine-pyrimethamine in the treatment of acute uncomplicated Plasmodium falciparum malaria in Nigerian children. Tran $R$ Soc. Trop Med Hyg 1997; 91: 58-62.

17. Sibley CH, Hyde JE, Sums PFG, Plore CV, Kublin JG, Mberu EK et al. Sulfadoxinepyrimethamine resistance in Plasmodium falciparum: what next? Trends Parasitol 2001; 17: 582-588.

18. Sowunmi A, Oduola AMJ. Viability of Plasmodium falciparum ex vivo: comparison of the effects of artemether and sulfadoxine-pyrimethamine. Eur J Clin Pharmacol. 1998; 54: 221-226.

19. Nwobodo N, Okonkwo PO. Comparative study on the efficacy of sulfadoxinepyrimethamine, amodiaquine and amodiaquine plus sulfadoxine-pyrimethamine combination in the treatment of acute uncomplicated malaria in Enugu State, Nigeria. Biomedical and Pharmacology Journal 2008; 1(2): 301-304.

20. Watkins WM, Woodrow C, Marsh K. Falciparum malaria: differential effects of antimalarial drugs on ex vivo parasite viability during the critical early phase of therapy. Am J Trop Med Hyg 1993; 49: 106-122.

21. Grellier P, Rigomier D, Schrevel J. In vitro induction of Plasmodium falciparum schizogony by the human high density lipoproteins (HDL). CR Acad Sci Paris III 1990; 311: 361-367.

22. Cutbert JA, Lipsky PE. Lipoproteins may provide fatty acids necessary for human lymphocyte proliferation by both low density lipoprotein receptor-dependent and independent mechanisms. J Biol Chem 1989; 264: 13468-13474. 
Table 1: Baseline Characteristics of Test and Control Groups

\begin{tabular}{|c|c|c|c|}
\hline Characteristics & Test & Control & p-Value \\
\hline Number of Patients & 30 & 30 & - \\
\hline Male: Female Ratio & $2: 3$ & $2: 3$ & - \\
\hline Mean Age (Range: $16-65$ years) & $41.7 \pm 2.5$ & $38.2 \pm 2.6$ & $\mathrm{p}>0.05$ \\
\hline Mean Weight (Range: 43-92 kg) & $60.8 \pm 3.7$ & $63.4 \pm 3.2$ & $\mathrm{p}>0.05$ \\
\hline Mean Temperature (Range: $37.8-39.2^{\circ} \mathrm{C}$ ) & $39.2 \pm 1.9$ & $38.0 \pm 1.6$ & $\mathrm{p}>0.05$ \\
\hline $\begin{array}{l}\text { Mean Parasite Density (Range: 1260- } \\
21500 / \mu \mathrm{L})\end{array}$ & $9635 \pm 791$ & $8791 \pm 750$ & $\mathrm{p}>0.05$ \\
\hline Mean Hemogram (Range: $4.2-11.5 \mathrm{~g} / \mathrm{dL})$ & $8.2 \pm 1.3$ & $7.9 \pm 1.0$ & $\mathrm{p}>0.05$ \\
\hline $\begin{array}{l}\text { Mean WBC Total (Range: } 3000-11700 x \\
10^{9} / \mathrm{L} \text { ) }\end{array}$ & $6955 \pm 452$ & $7470 \pm 455$ & $\mathrm{p}>0.05$ \\
\hline $\begin{array}{l}\text { Mean Alanine Transaminase } \\
\text { (Range: } 7.8-31.2 \mathrm{U} / \mathrm{L} \text { ) }\end{array}$ & $14.8 \pm 3.5$ & $15.6 \pm 4.3$ & $\mathrm{p}>0.05$ \\
\hline $\begin{array}{l}\text { Mean Aspartate Transaminase } \\
\text { (Range: } 13.7-28.4 \mathrm{U} / \mathrm{L} \text { ) }\end{array}$ & $15.8 \pm 4.6$ & $17.7 \pm 4.8$ & $\mathrm{p}>0.05$ \\
\hline $\begin{array}{l}\text { Mean Alkaline Phosphatase (Range: 45.2- } \\
\text { 110.7U/L) }\end{array}$ & $85.1 \pm 7.9$ & $79.6 \pm 7.7$ & $\mathrm{p}>0.05$ \\
\hline Mean Total Bilirubin (Range 4.3-13.8 $\mu \mathrm{mol} / \mathrm{L})$ & $8.7 \pm 1.4$ & $9.1 \pm 1.4$ & $\mathrm{p}>0.05$ \\
\hline
\end{tabular}

Table 2: Mean Treatment Failure in the Test and Control Groups

\begin{tabular}{lccc}
\hline Treatment Parameters & Test & Control & P-Value \\
\hline ETF $(\%)$ & $9.2 \pm 0.26$ & $20.5 \pm 0.26$ & $\mathrm{p}<0.05$ \\
LTF $(\%)$ & $10.3 \pm 0.15$ & $23.6 \pm 0.22$ & $\mathrm{p}<0.05$ \\
ACPR $(\%)$ & $77.5 \pm 0.59$ & $55.9 \pm 0.49$ & $\mathrm{p}<0.05$ \\
& & & \\
\hline
\end{tabular}

ETF: Early Treatment failure

LTF: Late Treatment failure

ACPR: Adequate Clinical and Parasitological Response 
Table 3: Mean clinical response in the test and control groups

\begin{tabular}{lccc}
\hline Clinical Parameters & Test & Control & p-Value \\
\hline PCT (Days) & $4.4 \pm 0.2$ & $9.8 \pm 0.2$ & $\mathrm{P}<0.05$ \\
FCT (hours) & $55.3 \pm 2.6$ & $87.4 \pm 4.5$ & $\mathrm{P}<0.05$ \\
CCR $(\%)$ & $82.3 \pm 0.8$ & $60 \pm 1.3$ & $\mathrm{P}<0.05$ \\
RR $(\%)$ & $19.1 \pm 0.14$ & $39 \pm 0.22$ & $\mathrm{P}<0.05$ \\
\hline
\end{tabular}

PCT: Parasite Clearance Time

FCT: Fever Clearance Time

CCR: Clinical Clearance Time

RR: Recrudescence Rate 


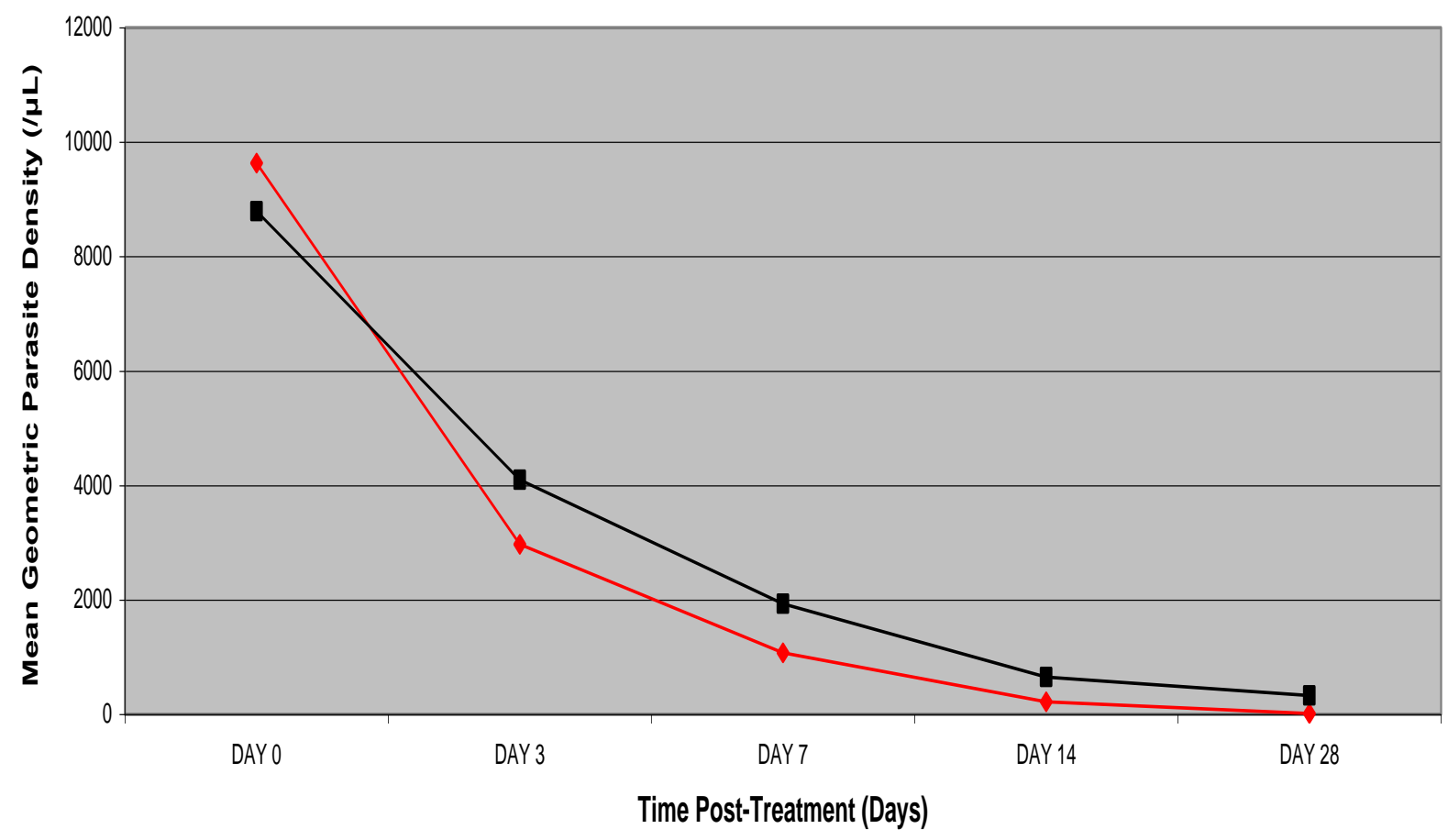

-Test (Sulfadoxine-Pyrimethamine and Simvastatin) $\quad \rightarrow$-Control (Sulfadoxine-Pyrimethamine Alone)

Figure: Depicts linear graphical representation of the progressive decline in the level of parasitemia in both test and control groups treated with sulfadoxine-pyrimethamine. It revealed mean geometric parasite density of $9635 / \mu \mathrm{L}, 2971 / \mu \mathrm{L}, 1076 / \mu \mathrm{L}, 217 / \mu \mathrm{L}$ and $13 / \mu \mathrm{L}$ for days $0,3,7,14$ and 28 respectively in the test group. This is as compared to the mean geometric parasite density of $8791 / \mu \mathrm{L}, 4098 / \mu \mathrm{L}, 1934 / \mu \mathrm{L}, 650 / \mu \mathrm{L}$ and $326 / \mu \mathrm{L}$ reported in 